\title{
Radiological assessment of irreducible posterolateral knee subluxation after dislocation due to interposition of the vastus medialis: a case report
}

\author{
Emilie Paulin • Sana Boudabbous • \\ Jean-Damien Nicodème $\cdot$ Daniel Arditi • \\ Christoph Becker
}

Received: 29 July 2014 / Accepted: 8 December 2014 / Published online: 6 January 2015

(C) ISS 2015

\begin{abstract}
Knee dislocation is a serious and relatively uncommon traumatism that every emergency room is supposed to diagnose and treat rapidly. Most of the time these dislocations reduce spontaneously or with closed reduction. If a subluxation persists, an incarceration of soft tissue in the joint must be suspected. Irreducible knee subluxations after dislocation are rare entities better described in the orthopaedic than in the radiological literature. However, the initial radiological assessment is an important tool to obtain the correct diagnosis, to detect neurovascular complications, and to plan the most suitable treatment. In cases of delayed diagnosis, the functional prognosis of the joint and even the limb may be seriously compromised primarily because of vascular lesions. Thereby, vascular imaging is essential in cases of dislocation of the knee, and we will discuss the role of angiography and the more recent use of computed tomography angiography or magnetic resonance angiography. Our patient presented with an irreducible knee subluxation due to interposition of the vastus medialis, and we will review the classical clinical presentation and 'do not miss' imaging findings on conventional radiography, computed tomography angiography, and magnetic resonance imaging. Finally, we will also report the classical imaging pathway indicated in knee dislocation, with a special emphasis on the irreducible form.
\end{abstract}

Keywords Irreducible knee dislocation · Vastus medialis

E. Paulin $(\bowtie) \cdot$ D. Arditi $\cdot$ C. Becker

Hôpitaux Universitaires de Genève, Service de Radiologie, Rue

Gabrielle-Perret-Gentil 4, 1205 Geneva, Switzerland

e-mail: emilie.paulin@bluewin.ch

S. Boudabbous $\cdot$ J.-D. Nicodème

Hôpitaux Universitaires de Genève, Service d'Orthopédie, Geneva,

Switzerland

\section{Introduction}

Posttraumatic irreducible knee subluxations after dislocation are rare and severe entities that require early care for both initial assessment of lesions and planning of orthopaedic treatment $[1,2]$. Moreover, sequelae after knee dislocation significantly reduce the functional prognosis of the joint and consequently the quality of life for patients [3]. Their irreducibility is most often secondary to incarceration of capsular, ligamentous, or muscular structures within the joint space [4]. In the case of posterolateral dislocation, the structures usually involved are the medial meniscus, capsule, medial collateral ligament, and vastus medialis [4].

Neurovascular lesions are also frequent and essential to depict [3]. Arterial injury management depends on the severity of the lesion and the different treatments range from nonsurgical options to bypass vascular surgery [5]. An untreated popliteal lesion increases the risk of amputation and must be detected ant treated rapidly [6]. Damage to the common peroneal nerve must be evaluated by clinical examination and by electromyography in the medium term if any doubt persists [7].

We report one case of such irreducible posterolateral subluxation after dislocation of the knee due to interposition of the vastus medialis and review the classical clinical aspect and 'do not miss' imaging findings on conventional radiography, computed tomography angiography, and MRI (magnetic resonance imaging). Knowledge of the imaging pathway and features is necessary to correctly diagnose these cases and identify neurovascular complications that significantly worsen the functional outcome.

\section{Materials and methods}

Our patient is a 54-year-old male with no relevant past medical or surgical history who fell and twisted his right knee 
while skiing. Pain and total impotence resulting from trauma required the patient to be transferred to our hospital for the management of a suspected posttraumatic dislocation of the right knee.

The clinical examination was immediately performed in the emergency room by an orthopaedic surgeon. Then the patient sequentially underwent anteroposterior and lateral conventional radiographs, computed tomography angiography of the lower limbs, and MRI of the right knee. The patient was finally treated by open surgery for reduction and stabilisation.

\section{Results}

On arrival, the patient presented considerable swelling of the right knee, with medial cutaneous incarceration (Fig. 1), known as a 'dimple sign' [4]. The mobility was limited with knee flexion at $50^{\circ}$ and impossibility of flexion/extension movements. There was no detectable neurovascular disorder. Peripheral pulses, skin colour, and local temperature were symmetric in both legs. Sensibility was normal and motor

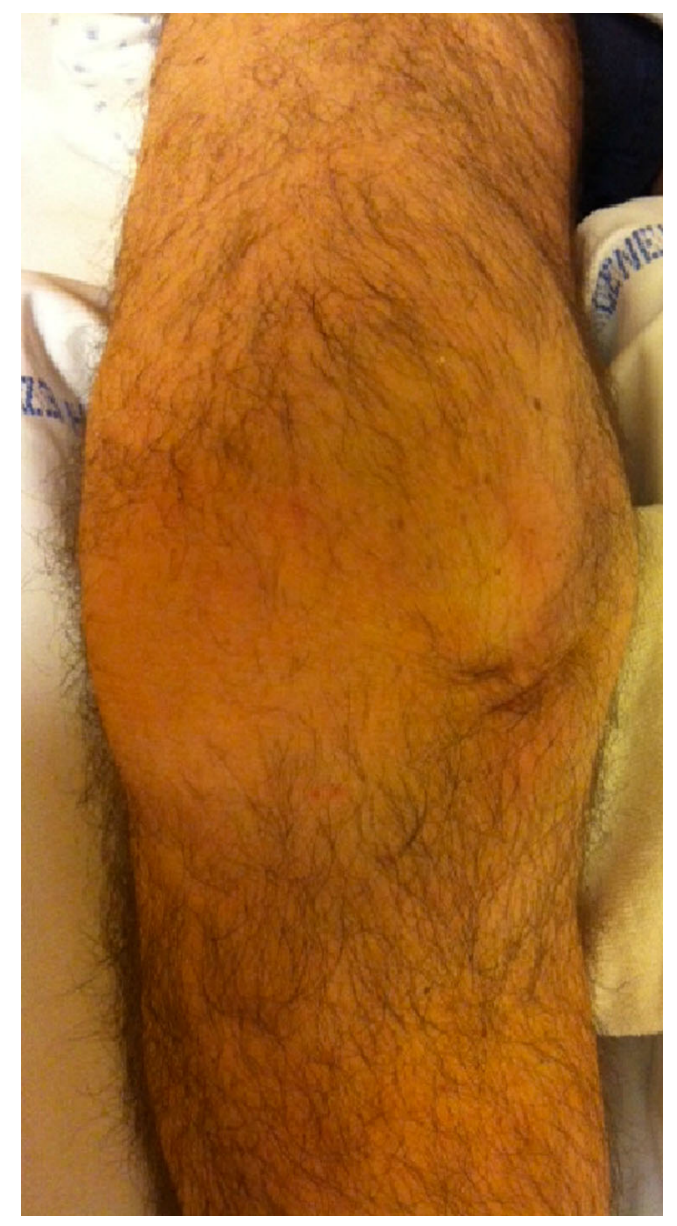

Fig. 1 A 54-year-old male suspected of posttraumatic irreducible knee subluxation after dislocation. Preoperative photography illustrates the characteristic 'dimple sign' function seemed to be limited only by the flexion and pain, without a suspected neurological deficit.

Anteroposterior and lateral conventional radiographs (Fig. 2) showed a discrete avulsion of the lateral femoral condyle, suggesting a ligamentous tear of the lateral collateral ligament, an enlargement of the medial femorotibial space, an important effusion, and a medial soft tissue attraction.

Computed tomography angiography with contrast injection in the arterial phase (Fig. 3) excluded arterial injury associated with the dislocation. Finally, magnetic resonance imaging (Figs. 4, 5 and 6) proved vastus medialis partial incarceration within the intercondylar notch, associated with medial femorotibial trapping of the medial patellofemoral ligament, capsule, and subcutaneous tissue. Associated ligamentous tears included complete rupture of the anterior and posterior cruciate ligaments as well as internal and external collateral ligaments.

Less than $24 \mathrm{~h}$ after the patient's arrival, all of the imaging described above was performed and the patient underwent surgical reduction by parapatellar medial arthrotomy (Fig. 7). At the same time, stabilisation of the knee was provided by reinsertion of the medial patellofemoral ligament, posterior oblique ligament, and medial collateral ligament. Cruciate ligament surgery was not undertaken for this patient.

The patient evolved favourably and was discharged from the orthopaedic unit 15 days after surgery. Consultation controls with oral interview and clinical examination at 6 weeks, 3 months, and 6 months confirmed this favourable evolution in terms of healing, pain, joint stability, and passive and active range of motion. The patient resumed his normal professional and extra-professional activities less than 1 year after the accident.

\section{Discussion}

Knee dislocation is defined as a complete loss of femorotibial congruence and subluxation as a disruption, with parts of the articular surfaces remaining in contact $[3,7]$. The classifications are based on the tibial displacement or on the pattern of ligament injury. The tibial displacement may be anterior (40\%), posterior (33\%), lateral (18\%), medial (4\%), or rotatory $(5 \%)[4,8]$. Later, Schenck et al. [9] proposed a more biomechanical classification, using the pattern of ligament injury, and this classification has finally been modified by Wascher et al. [10] to include associated lesions as vascular, neurological, or osseous. This modified Schenck classification is the most commonly used in clinical practice.

Posttraumatic knee dislocations are rare and generally reduced spontaneously or by a closed method. Most of the time, their irreducibility is associated with interposition of soft tissue structures such as the medial meniscus, capsule, retinaculum, and vastus medialis in posterolateral dislocations 
Fig. 2 Anteroposterior (a) and lateral (b) conventional radiographs show a discrete fracture of the lateral femoral condyle (white arrow), enlargement of the medial femorotibial space (doubleheaded white arrow), a medial soft tissue attraction (yellow arrow), and an important effusion (red arrow)
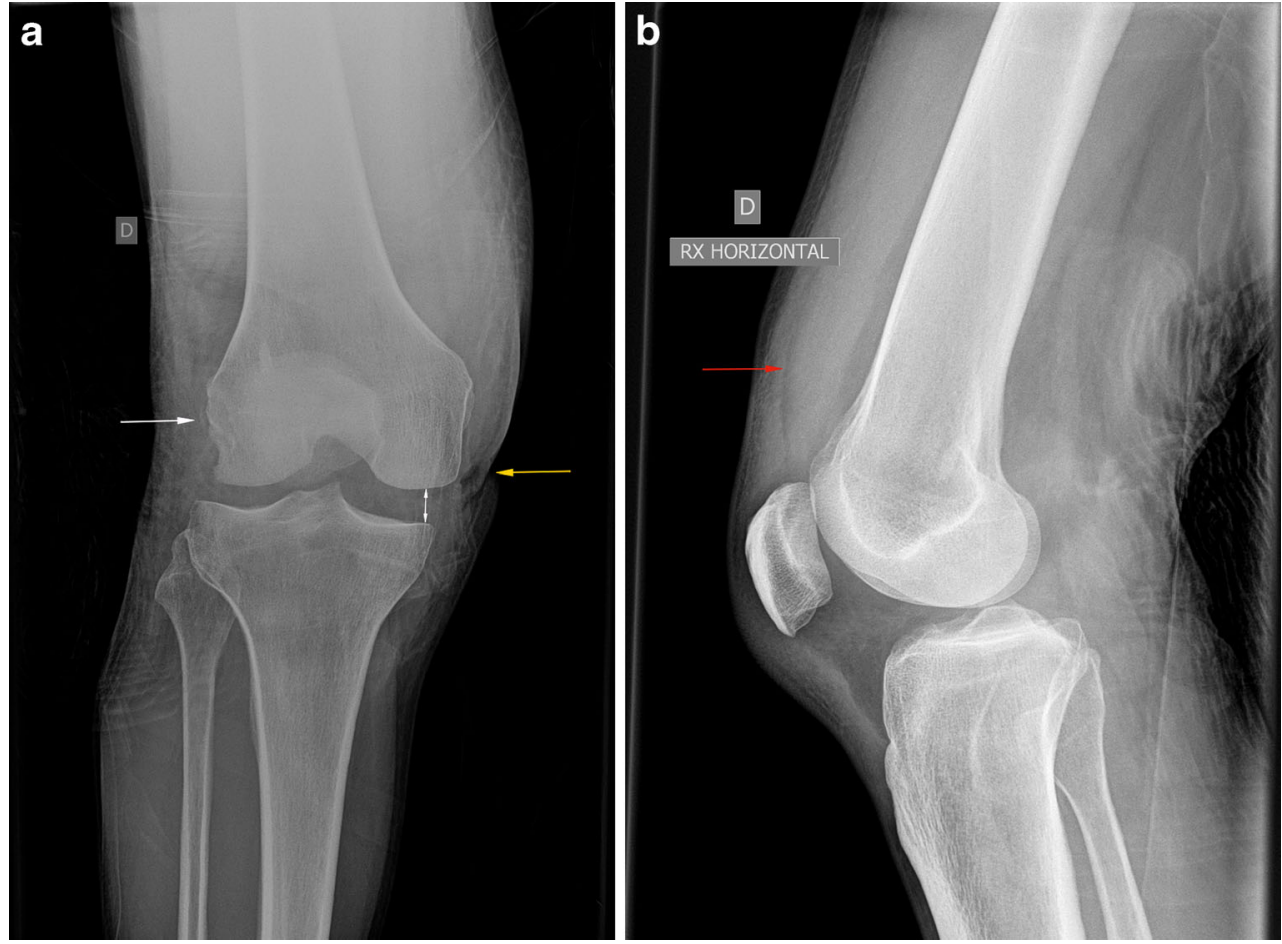

[11-13]. The mechanism implicated in a posterolateral dislocation can be high, low, or sometimes very low energy trauma $[3,14]$ and is usually secondary to valgus and internal rotation of the tibia.

Clinically, the most specific sign of an irreducible subluxation after dislocation seems to be the 'dimple sign' suggesting the interposition of medial structures [4]. However, clinical evaluation of knee dislocation patients is difficult most of the time because of the pain and, eventually, other lifethreatening injuries. American College of Radiology criteria summarise the clinical parameters necessitating realisation of radiographic views of the knee after acute trauma as joint effusion within $24 \mathrm{~h}$, palpable tenderness over the fibular head or patella, inability to walk or bear weight, inability to flex the knee to $90^{\circ}$, and altered mental status [15]. Radiographic signs of irreducible subluxation after dislocation are subtle and include misalignment of the femur and tibia as well as the enlargement of the femorotibial joint without gross dislocation. These signs as well as the radiographic 'dimple sign' corresponding to the clinically visible attraction of the soft tissues can easily be missed and should be looked for carefully in case of clinical suspicion.
Fig. 3 Computed tomography angiography with $3 \mathrm{D}$

reconstructions excludes injury of the popliteal artery
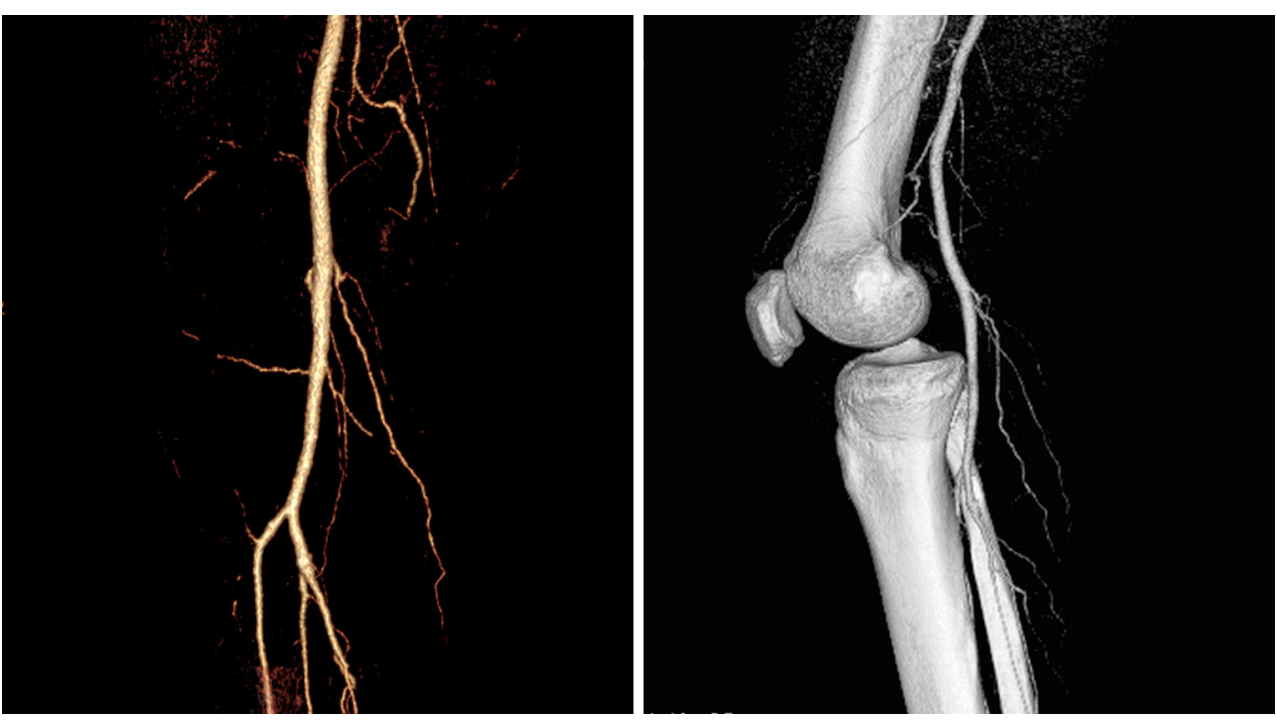

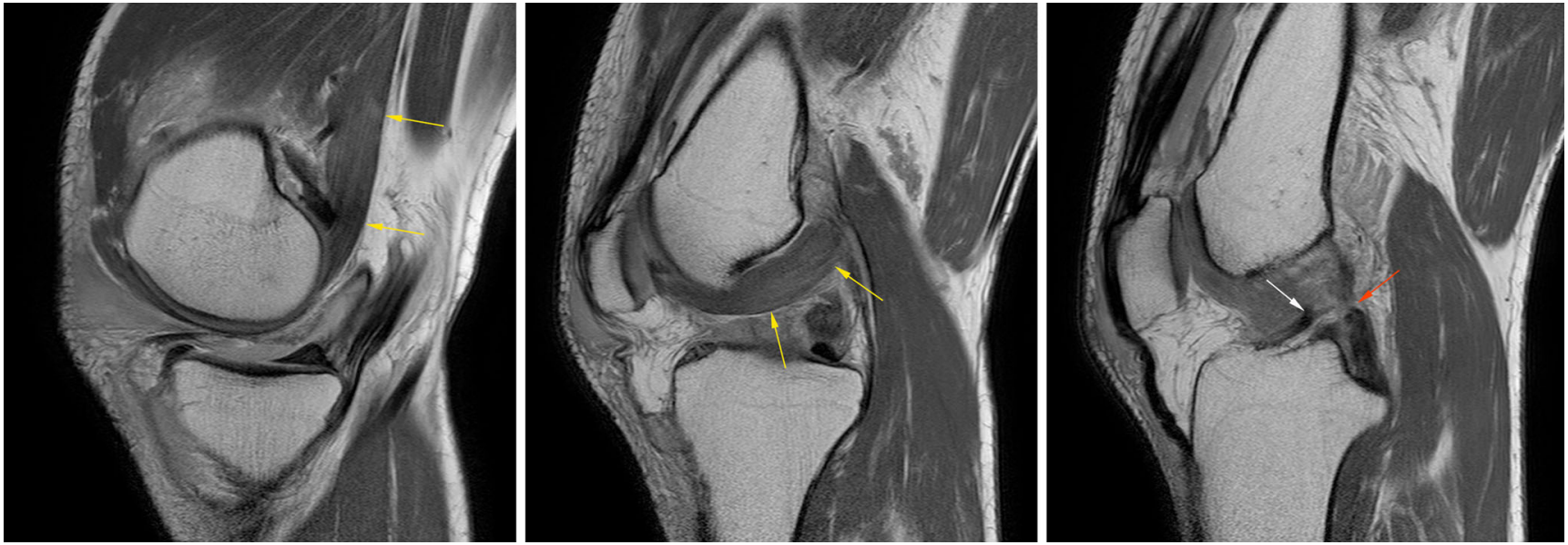

Fig. 4 Sagittalprotondensity-weightedimagesconfirmincarcerationof thevastusmedialismuscleintheintercondylarnotcharea(yellowarrows) and complete rupture of anterior (white arrow) and posterior (red arrow)

MRI for these patients was proved to be more accurate in the assessment of soft tissue damage, in particular ligamentous injuries [16]. Associated injuries of the menisci, cartilage, bone, and capsule or other soft tissue damage can also be easily described. The 'dimple sign' corresponding to the medial condyle buttonholing through soft tissues described clinically is shown by MRI on coronal views [17].

For any type of knee dislocation, vascular injury has to be quickly and carefully searched for due to the risk of amputation, which may increase to $86 \%$ in case of treatment delay of more than $8 \mathrm{~h}$ [6]. Arterial injury concerns the popliteal artery and is reported in about $30 \%$ of knee dislocations. This elevated rate is probably due to the poor collateral arterial pathway and to its proximal and distal fixed attachments above the knee at the adductor hiatus and below the joint behind the soleal arcade [7]. The artery may present any type of injury, from transient abnormality by kinking or timelimited occlusion to dissection, thrombosis, and transection. cruciate ligaments. Note the wrapping of the muscle around the distal femur

Physical examination must include observation of colour and temperature and palpation of the dorsalis pedis and posterior tibial arteries. The absence of pulses, presence of bruit or thrill, and presence of ischaemic signs or active haemorrhage seem to be $100 \%$ accurate for determining the need for surgical exploration. The ankle brachial index is another simple and noninvasive tool for assessing vascular perfusion. It corresponds to the ratio of the systolic ankle pressure to systolic arm pressure. An ankle brachial index of $>0.9$ was also proved to be $100 \%$ accurate in excluding vascular injury [18].

If this clinical assessment is clearly pathological, surgery is advocated, but the good practice for an asymptomatic patient remains controversial, since secondary clotting on an intimal lesion and a complete lesion with peripheral normal pulse were described [19-21]. Most authors consider that vascular imaging remains justified in this serious traumatic context [22-24], but the historical invasive angiogram is progressively being replaced by computed tomography angiography $[7,15$,
Fig. 5 Coronal T1 spin echoweighted image (a) and coronal short tau inversion recoveryweighted image (b) show tears of both internal (yellow arrow) and external (red arrow) collateral ligaments. Note the focal bone contusion (white arrow) corresponding to the fracture described on the conventional radiograph. The medial meniscus has a normal feature
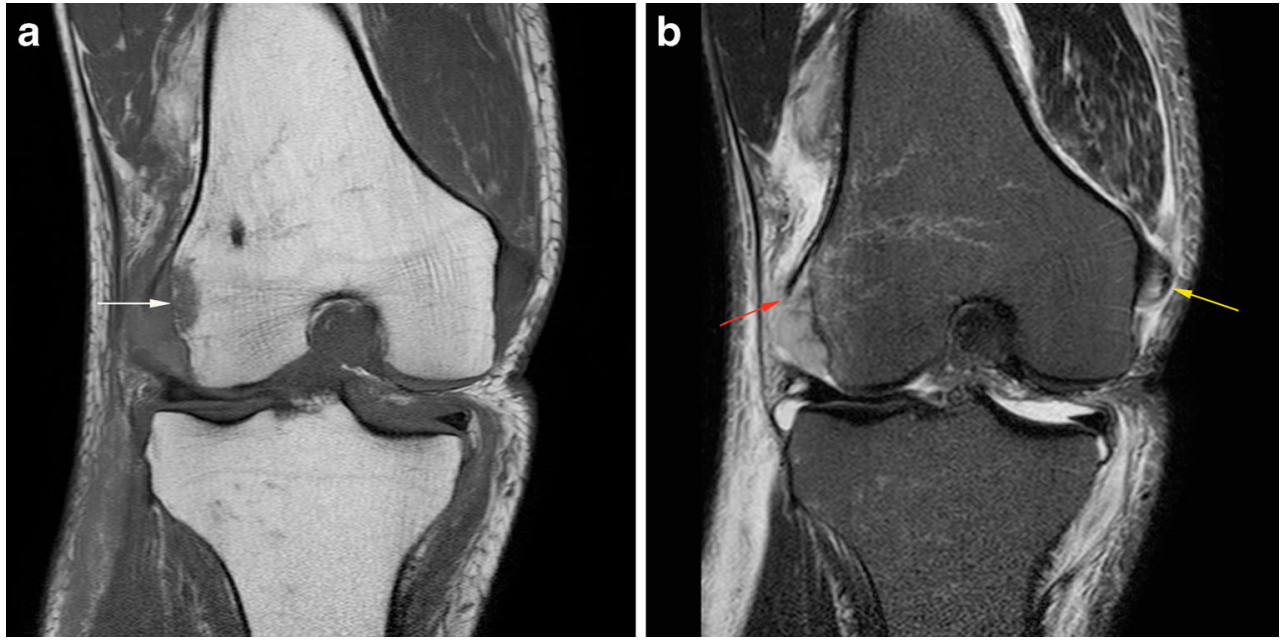


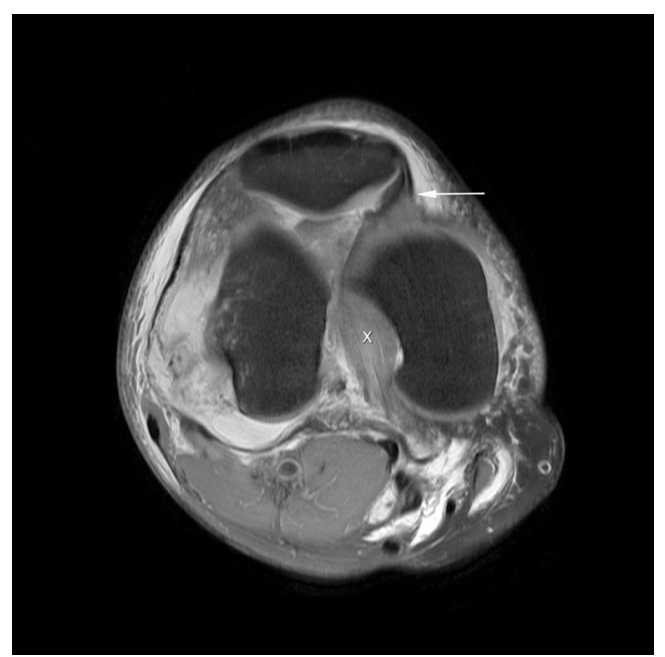

Fig. 6 Axial fat-suppressed proton density-weighted image with intercondylar trapping of the medial patellofemoral ligament (white arrow) and the vastus medialis (X)

25]. This technique decreases the rate of complication by its noninvasive feature and is demonstrated to be as accurate as arteriography in the diagnosis of traumatic arterial injuries [26, 27]. Moreover, knee dislocation may be associated with other injuries in case of polytraumatised patients, for whom exploration by computed tomography is better.

The advantage of magnetic resonance angiography is that the examination can be done at the same time as MRI for softtissue injury diagnosis and it correlates $100 \%$ with angiogram findings in multiple ligament knee injuries [28]. The problem with magnetic resonance angiography remains its accessibility and the long acquisition time, during which the patient must remain still. If ligament stabilisation is not considered, and if

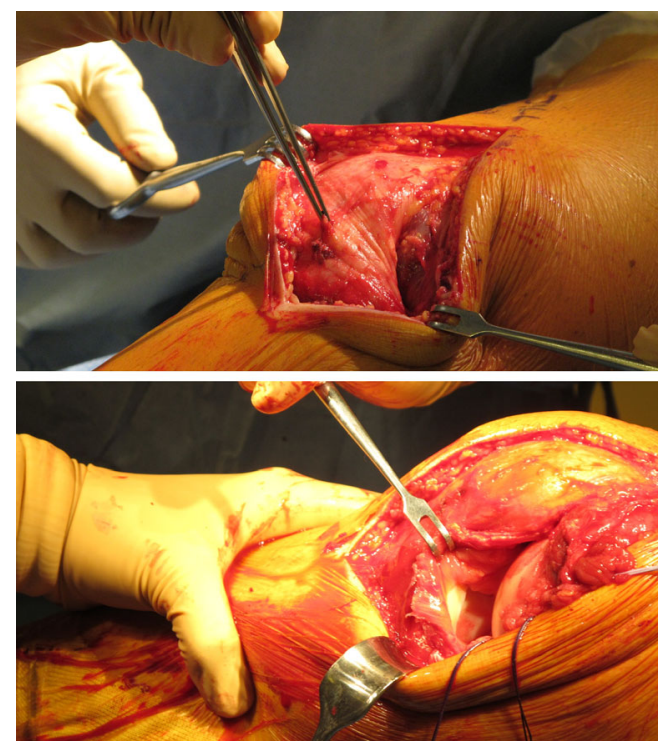

Fig. 7 Peroperative photographs. Medial parapatellar arthrotomy allows good visualisation of muscular incarceration the examination is not associated with MR angiography, MRI for the assessment of structural damage could be delayed. Finally, the current recommended approach is to propose immediate vascular noninvasive imaging by computed tomography angiography or magnetic resonance angiography.

Similarly, the common peroneal nerve is injured in 20 $30 \%$ of cases of knee dislocation [29] because of stretching or rupture caused by traction. The initial assessment is clinical and by MRI because electromyographic changes are not present in the first weeks after injury [7, 30].

In case of irreducibility, the patient requires prompt treatment by open surgery. After reduction, the treatment remains controversial but the literature describes better outcomes for surgical stabilisation of ligamentous tears compared with conservative treatment, while the timing of these reconstructions remains variable [31,32]. A recent prospective study [33] suggests that open dislocations and vascular disease should be surgically treated in emergency, without associated ligament reconstruction. In other cases, ligament stabilisation would be an option for patients under 60 years of age.

The irreducible knee subluxation after dislocation with interposition of musculo-ligamentous structures is rare but its clinical appearance and orthopaedic management are well described because of the functional risk to the joint and even the limb. Radiological assessment is also an important part of the management, in the diagnosis, description of implicated structures, and functional prognosis prediction. Radiographs are the first necessary step to diagnose subluxation and to exclude associated fractures. Vascular imaging is imperative in the management of these patients as the prognosis is closely related to the presence of vascular complications, and progressively, noninvasive imaging such as computed tomography and magnetic resonance angiography are replacing conventional angiography. MRI allows the study of vascular and soft tissues at the same time but is not readily available in all emergency centres.

Conflict of interest The authors declare that they have no conflict of interest.

\section{References}

1. Howells NR, Brunton LR, Robinson J, Porteus AJ, Eldridge JD, Murray JR. Acute knee dislocation: an evidence based approach to the management of the multiligament injured knee. Injury. 2011;42(11):1198-204.

2. Seroyer ST, Musahl V, Harner CD. Management of the acute knee dislocation: the Pittsburgh experience. Injury. 2008;39(7):710-8.

3. Eranki V, Begg C, Wallace B. Outcomes of operatively treated acute knee dislocations. Open Orthop J. 2010;4:22-30.

4. Durakbasa MO, Ulku K, Ermis MN. Irreducible open posterolateral knee dislocation due to medial meniscus interposition. Acta Orthop Traumatol Turc. 2011;45(5):382-6. 
5. Gray JL, Cindric M. Management of arterial and venous injuries in the dislocated knee. Sports Med Arthrosc Rev. 2011;19(2):131-8.

6. Green NE, Allen BL. Vascular injuries associated with dislocation of the knee. J Bone Joint Surgery Am Vol. 1977;59(2):236-9.

7. Walker RE, McDougall D, Patel S, Grant JA, Longino PD, Mohtadi NG. Radiologic review of knee dislocation: from diagnosis to repair. AJR Am J Roentgenol. 2013;201(3):483-95.

8. Kennedy JC. Complete dislocation of the knee joint. J Bone Joint Surgery Am Vol. 1963;45:889-904.

9. Schenck Jr RC. The dislocated knee. Instr Course Lect. 1994;43: 127-36.

10. Wascher DC, Dvirnak PC, DeCoster TA. Knee dislocation: initial assessment and implications for treatment. J Orthop Trauma. 1997;11(7):525-9.

11. Urguden M, Bilbasar H, Ozenci AM, Akyildiz FF, Gur S. Irreducible posterolateral knee dislocation resulting from a low-energy trauma. Arthrosc J Arthrosc Relat Surgery Off Publ Arthrosc Assoc North Am Int Arthrosc Assoc. 2004;20 Suppl 2:50-3.

12. Silverberg DA, Acus R. Irreducible posterolateral knee dislocation associated with interposition of the vastus medialis. Am J Sports Med. 2004;32(5):1313-6.

13. Kilicoglu O, Demirhan M. Irreducible posterolateral knee dislocation associated with interposition of the vastus medialis. Am J Sports Med. 2005;33(12):1931. author reply 1931.

14. Cinar M, Derincek A, Akpinar S. Irreducible dislocation of the knee joint: two-stage treatment. Acta Orthop Traumatol Turc. 2011;45(4): 280-3.

15. Tuite MJ, Daffner RH, Weissman BN, Bancroft L, Bennett DL, Blebea JS, et al. ACR appropriateness criteria $((\mathrm{R}))$ acute trauma to the knee. J Am Coll Radiol JACR. 2012;9(2):96-103.

16. Twaddle BC, Hunter JC, Chapman JR, Simonian PT, Escobedo EM. MRI in acute knee dislocation. A prospective study of clinical, MRI, and surgical findings. J Bone Joint Surgery Br Vol. 1996;78(4):573-9.

17. Harb A, Lincoln D, Michaelson J. The MR dimple sign in irreducible posterolateral knee dislocations. Skelet Radiol. 2009;38(11):1111-4.

18. Mills WJ, Barei DP, McNair P. The value of the ankle-brachial index for diagnosing arterial injury after knee dislocation: a prospective study. J Trauma. 2004;56(6):1261-5.

19. Alberty RE, Goodfried G, Boyden AM. Popliteal artery injury with fractural dislocation of the knee. Am J Surg. 1981;142(1):36-40.

20. Jones RE, Smith EC, Bone GE. Vascular and orthopedic complications of knee dislocation. Surgery Gynecol Obstet. 1979;149(4):554-8.
21. Collins HA, Jacobs JK. Acute arterial injuries due to blunt trauma. J Bone Joint Surgery Am Vol. 1961;43-A:193-7.

22. Boisrenoult P, Lustig S, Bonneviale P, Leray E, Versier G, Neyret P, et al. Vascular lesions associated with bicruciate and knee dislocation ligamentous injury. Orthop Traumat Surgery Res OTSR. 2009;95(8): 621-6.

23. McDonough Jr EB, Wojtys EM. Multiligamentous injuries of the knee and associated vascular injuries. Am J Sports Med. 2009;37(1): $156-9$.

24. Stannard JP, Sheils TM, Lopez-Ben RR, McGwin Jr G, Robinson JT, Volgas DA. Vascular injuries in knee dislocations: the role of physical examination in determining the need for arteriography. J Bone Joint Surgery Am Vol. 2004;86-A(5):910-5.

25. Shearer D, Lomasney L, Pierce K. Dislocation of the knee: imaging findings. J Spec Oper Med Peer Rev J SOF Med Prof. 2010;10(1): 43-7.

26. Fleiter TR, Mervis S. The role of 3D-CTA in the assessment of peripheral vascular lesions in trauma patients. Eur J Radiol. 2007;64(1):92-102.

27. Peng PD, Spain DA, Tataria M, Hellinger JC, Rubin GD, Brundage SI. CT angiography effectively evaluates extremity vascular trauma. Am Surg. 2008;74(2):103-7.

28. Potter HG, Weinstein M, Allen AA, Wickiewicz TL, Helfet DL. Magnetic resonance imaging of the multiple-ligament injured knee. J Orthop Trauma. 2002;16(5):330-9.

29. Bonnevialle P, Dubrana F, Galau B, Lustig S, Barbier O, Neyret P, et al. Common peroneal nerve palsy complicating knee dislocation and bicruciate ligaments tears. Orthop Traumat Surgery Res OTSR. 2010;96(1):64-9.

30. Cush G, Irgit K. Drop foot after knee dislocation: evaluation and treatment. Sports Med Arthrosc Rev. 2011;19(2):139-46.

31. Liow RY, McNicholas MJ, Keating JF, Nutton RW. Ligament repair and reconstruction in traumatic dislocation of the knee. J Bone Joint Surgery Br Vol. 2003;85(6):845-51.

32. Hirschmann MT, Meier MD CFA, Friederich NF. Long-term outcome of patients treated surgically for traumatic knee dislocation: does the injury pattern matter? Phys Sports Med. 2010;38(2):82-9.

33. Lustig S, Leray E, Boisrenoult $P$, Trojani C, Laffargue P, Saragaglia $\mathrm{D}$, et al. Dislocation and bicruciate lesions of the knee: epidemiology and acute stage assessment in a prospective series. Orthop Traumat Surgery Res OTSR. 2009;95(8):614-20. 